\title{
Improved Face Image Super-resolution with Restricted Patch-searching Area
}

\author{
Suhail Hamdan \\ Ritsumeikan University \\ 1-1-1 Noji-Higashi, Kusatsu-shi, \\ Shiga 525-8577, Japan. \\ Tomonori Izumi \\ Ritsumeikan University \\ 1-1-1 Noji-Higashi, Kusatsu-shi, \\ Shiga 525-8577, Japan. \\ t-izumi@se.ritsumei.ac.jp
}

\author{
Yohei Fukumizu \\ Ritsumeikan University \\ 1-1-1 Noji-Higashi, Kusatsu-shi, \\ Shiga 525-8577, Japan. \\ fukumizu@se.ritsumei.ac.jp
}

\author{
Hironori Yamauchi \\ Ritsumeikan University \\ 1-1-1 Noji-Higashi, Kusatsu-shi, \\ Shiga 525-8577, Japan.
}

\begin{abstract}
Recently, an example-based super-resolution (SR) method specifically for face image, which takes the correspondence of facial parts into consideration, was proposed. The method constructs a database of face-image patches with their positions from example images of normalized faces. Given a low-resolution (LR) image, the method generates an SR image by finding similar patches in the database and incorporating high-frequency component of the patches to the LR image. Since the method just divides an example image into patches, for example, $5 \times 5$ patches in five-pixels intervals, the SR process may misses the chance to have the patch of the finest position. The proposed method generates patches in one-pixel intervals to increase the chance. In order to cope with the massively increased size of the patch database, the proposed method restricts the area to search the patch candidates for each position. An experimental result shows the proposed method achieves better results than the previous in term of the peak signal-to-noise ratio (PSNR). Furthermore, the proposed method has reduced computational time by $90 \%$ compared to the previous.
\end{abstract}

\section{CCS Concepts}

- Computing methodologies Reconstruction

\section{Keywords}

example-based; face image; super-resolution; facial parts; patchsearching area; computational time.

\section{INTRODUCTION}

Face image super-resolution is adopted in many applications such

Permission to make digital or hard copies of all or part of this work for personal or classroom use is granted without fee provided that copies are not made or distributed for profit or commercial advantage and that copies bear this notice and the full citation on the first page. Copyrights for components of this work owned by others than ACM must be honored. Abstracting with credit is permitted. To copy otherwise, or republish, to post on servers or to redistribute to lists, requires prior specific permission and/or a fee.

Request permissions from Permissions@acm.org.

ICCSP 2019, January 19-21, 2019, Kuala Lumpur, Malaysia

(C) 2019 Association for Computing Machinery.

ACM ISBN 978-1-4503-6618-2/19/01 ..\$15.00

https://doi.org/10.1145/3309074.3309109 as video surveillance, face recognition and forensic investigation. For instance, face images from surveillance camera are frequently too poor in quality since most cameras record low-resolution (LR) frames due to storage constraint. Therefore, it is necessary to enhance the LR images into a clear high-resolution (HR) image using super-resolution techniques in advance so that we can extract useful information from the images.

Various methods [1-5] for image super-resolution have been introduced so far to reconstruct a high-resolution (HR) image from a LR image. One of them is example-based method or also known as learning-based approach [6-12], which is well adopted as a significant technique for face image super-resolution since it uses a collection of specific kinds of images as their dictionary. Example-based method utilizes external information from a set of training LR and HR image pairs, where a patch within an observed LR image is extracted and searched within the training set to estimate suitable HR patch that reconstructs the HR image. A learning model was employed to select suitable patch candidates based on their texture similarity and compatibility between adjacent patches in the image.

Recently, a constructive face images SR method, which takes into consideration the correspondence of facial parts, has been proposed [13]. The method utilizes a set of normalized human face images as training database, where facial parts can be estimated according to patch's original position in image. The nearer the position of training-patch to a currently observed patch's position, the higher the facial-parts compatibility. Their learning model selected patch candidates based on both texture similarity and facial parts' compatibility, i.e. using eye patches for eyes part and nose patches for nose part, according to a given weighting factor. This method gives better resulting HR image in terms of peak signal-to-noise ratio (PSNR) compare to the conventional method, i.e. Freeman et al.'s method [12].

However, since the method depends on patches' position or coordinate, patches from each image in database must also be divided in a manner of a grid map, i.e. if patch size is $5 \times 5$, patches should be cut with interval of five pixels vertically and horizontally. This way of patch-division consequently reduces diversity of patches' texture patterns from an image since some 

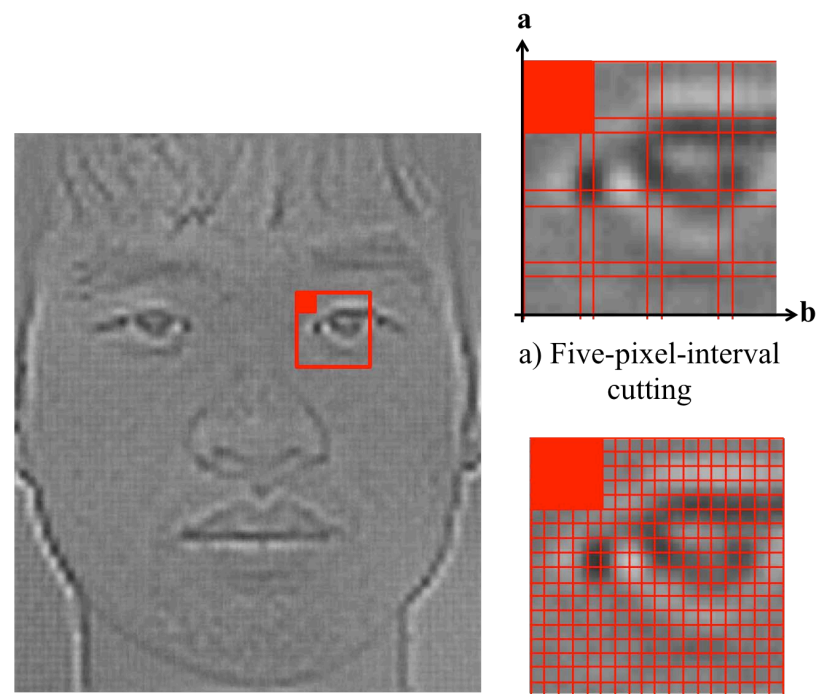

a) Five-pixel-interval cutting

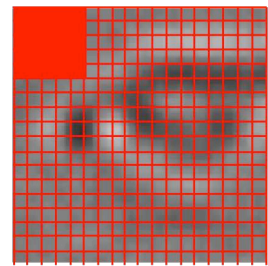

b) One-pixel-interval cutting

Figure 1. Patches generation method.

texture patterns may had been overlooked compare to pixel-bypixel cutting style. Figure 1 shows an example of comparison between both types of patch-division method, where colored box is an example of patch within a face image. Figure 1(a) presents the patch-by-patch dividing method with patch's coordinate $(a, b)$ with one pixel overlap border, while Figure 1(b) presents a fine way of patch division (pixel-by-pixel). We can observe that onepixel-interval cutting method gives more fine patches compare to patch-by-patch cutting. The number of patches from (b) is about 15 times more than the number of patches extracted from (a).

This paper proposed a method that utilizes an improved patchsearching algorithm, which is not using patch-position information and compatible with the one-pixel-interval patch cutting method. The proposed method restricts a specific patchsearching area around the targeted facial part to find patch candidates, in order to ensure that patches were selected by taking facial parts into consideration and cope with the massively increased size of the patch database. We employ a currently observed patch's position information as pinpointed position and specified a certain range of searching area nearby. In other words, we have liberalized the way of patch-division to get much better choice of patch's position or texture, but on the other hand restrict patch-searching area to ensure that patch candidates were selected by taking facial parts into consideration. Our aim is that the proposed method obtains better resulting image since more detailed patches were observed.

Let patch-searching area's range is $(2 s+1)^{2}$ in pixels, we demonstrated our method onto a set of 30 LR face images with different size of searching area, i.e. s was set as 3, 5, 10, 15, 20, 30 , and 40 to observe their performance. The size of patch was $5 \times 5$. Average PSNR for the 30 resulting HR images were observed. The results show that the proposed method gives better resulting PSNR than the previous method.

Furthermore, typically the wider the size of searching area the better the results, since more number of patches were observed, but our experiment results show differently. The resulting PSNRs show that when searching area, s was larger than 20, the PSNRs value decreased. We found that even if a selected patch has higher similarity of LR texture (middle-frequency component), it does not necessarily give plausible HR texture (high-frequency component) for an observed LR patch. Therefore, by restricting patch-searching area to ensure that patches were selected from a targeted facial part, the method increases the possibility of selecting plausible correspondence HR patch. On top of that, since our method searches within a limited number of patches during patch-candidates selection, computational time was considerably reduced by approximately $90 \%$ compare to the previous method.

The rest of the paper is organized as follows. Section 2 describes the algorithms of the example-based methods. Section 3 elaborates the learning model for recent method that takes into account correspondence of facial parts. Our proposed method is described in Section 4, and a conclusion is given at the end of the paper.

\section{EXAMPLE-BASED METHOD}

Example-based method is an SR technique that utilizes external information from a set of a large volume of training image pairs of LR and HR images. Compared to conventional interpolation methods that only use information from input images, the method enables new information to be predicted as HR texture details.

Example-based algorithms basically consists of two phases, i.e., 1) a database construction phase that extracts patch pairs from both LR and HR training images, and then stores them as training patches into database, and 2) a super-resolution phase that runs a learning model to reconstruct HR images by searching suitable patches in the database that are best matched to the input image patches.

\subsection{Database Construction}

A collection of high-resolution images is used to construct a training database. We restrict use of the same kinds of images to obtain better probability of similarity among textures. All HR images are downscaled, typically one-quarter the total number of pixels to create LR training images. After that, we initially upscale the LR images back into original size of HR using an analytic interpolation, such as Lanczos. These initial HR images are typically blurry due to the loss of texture during the process.

We preprocess the training images in pair (initially upscaled LR and original HR images) to extract high-frequency information so that only textures are being observed. We apply a Gaussian filter to the upscaled LR images to extract their textures (middlefrequency component) and store them into database, Po, as outlined in Figure 2. While textures from the original HR images are extracted by subtracting them with the initially upscaled LR images, then we store them as patches (high-frequency component) in database, Pc.

\subsection{Image Super-Resolution Process}

Freeman et al. implemented a Markov random field (MRF) network as learning model to estimate plausible texture for an output HR image [12]. The MRF network statistically models the spatial relationship between input image patches and estimated training patches in database, and between neighboring highresolution patches.

Figure 3 illustrates the structure of the MRF network where each circle represents a network node and each line indicates the spatial relationship and statistical dependencies between nodes. The nodes $Y(i, j)$ represents the LR patches at the position $(i, j)$ in the observed image. The nodes X(i,j) represents the LR patches in the database whose corresponding HR patches are used to reconstruct HR image. 


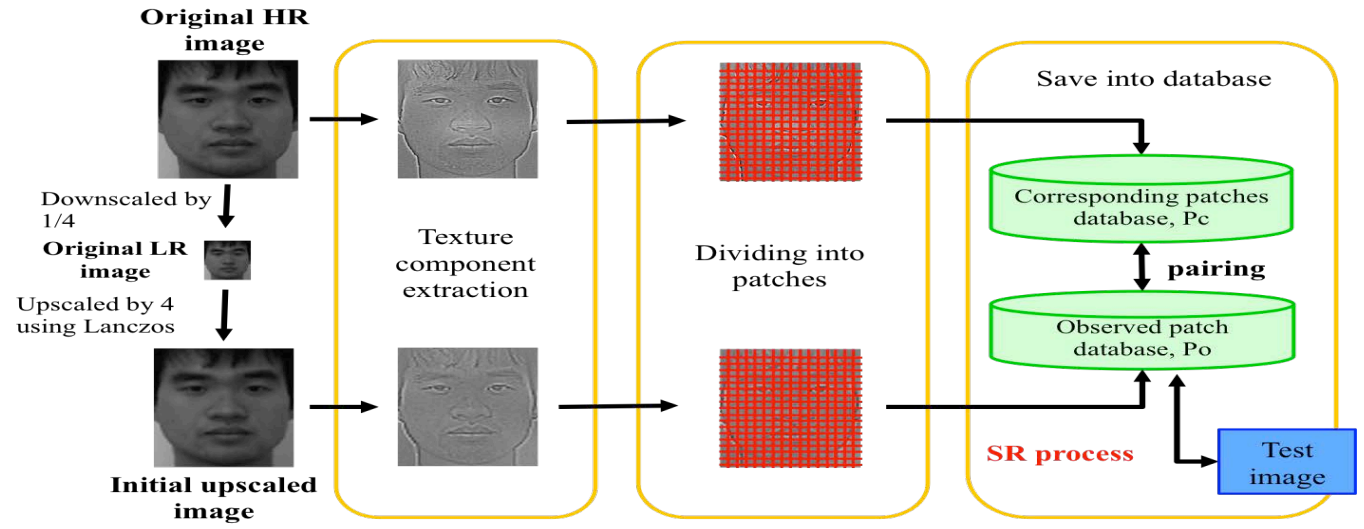

Figure 2. Database construction phase.

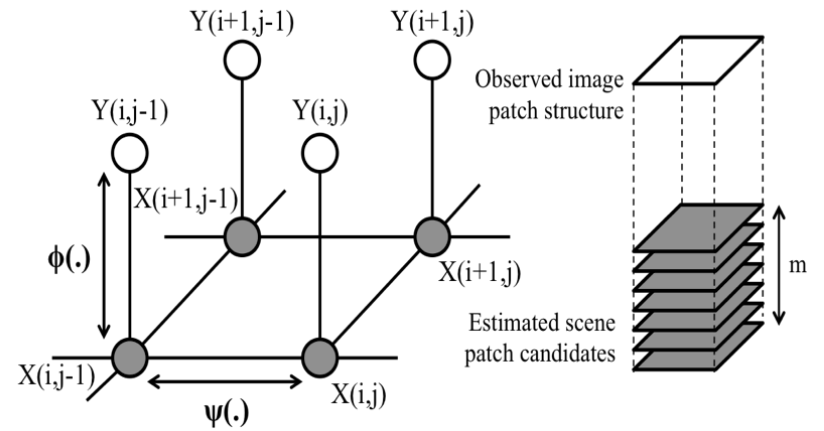

Figure 3. MRF-network-based learning model.

The dependency between nodes are represented by two compatibility functions, $\phi($.$) and \psi($.$) . For a position (\mathrm{i}, \mathrm{j})$ in the MRF network, $\phi[X(i, j), Y(i, j)]$ represents the compatibility between the observed patch $Y(i, j)$ and the training patch $X(i, j)$. For a position $(\mathrm{i}, \mathrm{j})$ and its adjacent position $(\mathrm{u}, \mathrm{v})$, the function $\psi[\mathrm{X}(\mathrm{i}, \mathrm{j}), \mathrm{X}(\mathrm{u}, \mathrm{v})]$ represents the compatibility of the common border between the estimated patches $X(i, j)$ and $X(u, v)$. The joint probability over $\mathrm{X}($.$) and \mathrm{Y}($.$) is defined as$

$$
\begin{aligned}
P(X \mid Y)= & \prod_{i j} \phi[X(i, j), Y(i, j)] \\
& \times \prod_{i j,(u, v) \in N B(i, j)} \psi[X(i, j), X(u, v)],
\end{aligned}
$$

where $\mathrm{NB}(\mathrm{i}, \mathrm{j})$ denotes the neighbors of $\mathrm{X}(\mathrm{i}, \mathrm{j})$ in the MRF network. A number of patch candidates for $\mathrm{X}(\mathrm{i}, \mathrm{j})$ are previously selected based on the $\phi($.$) . The number of patch candidates is$ given as a constant parameter $\mathrm{m}$.

To specify the $\phi($.$) , we impose a similar quadratic penalty on$ differences between the observed input image patch, $Y(i, j)$, and the patch candidates found from the training set, $X(i, j)$.

$$
\phi[X(i, j), Y(i, j)]=\exp \left\{-\frac{d[X(i, j), Y(i, j)]}{2 \sigma_{1}}\right\}
$$

$\mathrm{d}($.$) is the distance of the two matrices (or vectors) and \sigma_{1}$ is a constant parameter.

To specify the $\psi($.$) , we sample the input image's patches so that$ they overlap with each other by one or more pixels, as shown in Figure 4 . The border compatibility, $\psi($.$) is defined as$

$$
\psi[X(i, j), X(u, v)]=\exp \left[-\frac{d(p, q)}{2 \sigma_{2}}\right],
$$

where $p(q)$ is the vector of pixels of the overlap region in patch $\mathrm{X}(\mathrm{i}, \mathrm{j})\left(\mathrm{X}(\mathrm{u}, \mathrm{v})\right.$, respectively) and $\sigma_{2}$ is a constant parameter.

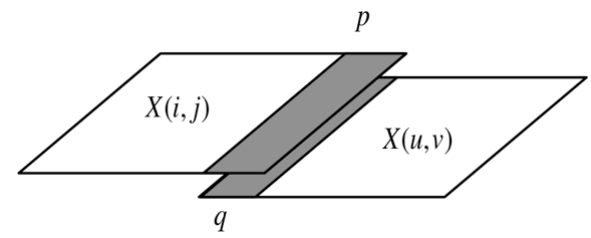

Figure 4. Overlap region between adjacent patches.

Given an initial set of patch candidates for $\mathrm{X}($.$) , the method$ iterates to change each candidate for $X(i, j)$ in turn to improve $\mathrm{P}(\mathrm{X} \mid \mathrm{Y})$ greedily until no improvement is observed. The HR patches corresponding to the finally chosen candidates for $\mathrm{X}(\mathrm{i}, \mathrm{j})$ forms the estimated texture (high-frequency component). The method combines the initially upscaled LR image and the estimated texture component to obtain a restored HR image.

\section{FACE IMAGE SUPER-RESOLUTION METHOD}

Recently, a method specifically for face-image super-resolution was proposed. The underlying idea is to estimate suitable patches for HR image's reconstruction by not only based on texture similarity, but also considering their facial parts, i.e. using eye patches for eyes and nose patches for nose. The learning model was modified from Freeman et al.'s so that it takes the correspondence of facial parts into account during patchcandidates selection. For this purpose, the method utilizes a set of normalized human face images as training database, where face feature points are approximately in closer position in every image. Therefore, facial parts can be estimated according to patch's original position in image, where distance between currently observed test image's patch and estimated training patch represents facial-parts compatibility in the learning model.

Let the facial-parts compatibility function is $\lambda($.$) , the function is$ defined as

$$
\lambda[X(i, j)]=\exp \left[-\frac{\ell}{2 \sigma_{3}}\right]
$$


where $\ell$ is Euclidean distance from original position of the patch $\mathrm{X}(\mathrm{i}, \mathrm{j})$ in the training face image to the observed position $(\mathrm{i}, \mathrm{j})$ in the restored image, and $\sigma_{3}$ is a constant parameter. Hence, the joint probability of $\mathrm{X}($.$) under the condition of \mathrm{Y}($.) is an extension of Equation 1 as defined in Equation 5.

$$
\begin{aligned}
P(X \mid Y)= & \prod_{i j} \phi[X(i, j), Y(i, j)] \\
& \times \prod_{i j,(u, v) \in N B(i, j)} \psi[X(i, j), X(u, v)] \\
& \times \prod_{i j} \lambda[X(i, j)]
\end{aligned}
$$

\subsection{Normalized Face Image Database Construction}

We start from a collection of normalized high-resolution face images for database. The normalized training images have the same size or ratio of facial features, where facial-feature points (e.g., eye, nose, mouth, chin, and face boundary lines) in each image are at approximately closer positions.

Figure 5 illustrates a patch-database construction process for a set of normalized face images. Since the learning model already includes $\lambda($.$) , it is unnecessary to categorize the patches into$ multiple databases based on facial parts. The main characteristic of the database is each patch's original position in the training images, $(\mathrm{k}, 1)$, as shown in Figure 5, will be observed during superresolution process. $m$ refers to the number of available training images in database.

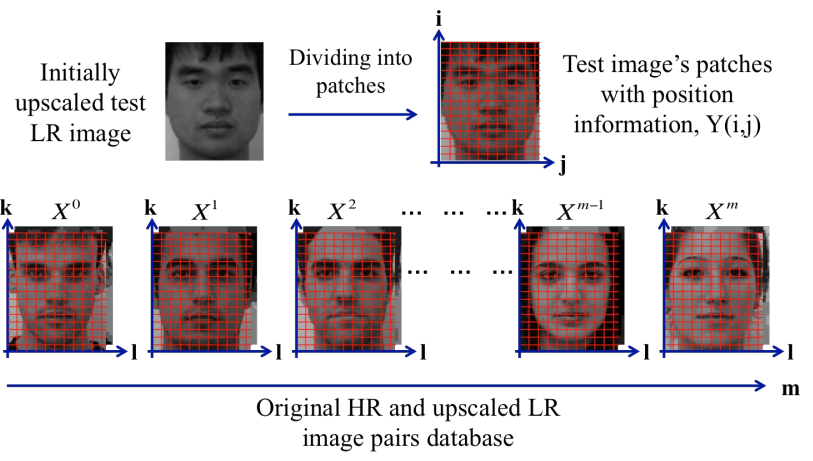

Figure 5. Coordinate-based patch database construction.

Same as the process shown in Figure 2, we degrade the training images by downsampling them into LR images under certain scaling factor, typically one-quarter the number of original pixels in each dimension (1/16 the total number of pixels). We then resample it back into the original size to create an initial upscaled image. Then, we filter out the lowest frequency component on both original HR and initially upscaled images to retain only their texture component. Both components are then divided into patches in a way where both patches correspond to each other. Patches were divided in a manner of five-pixels intervals as shown in Figure 1(a) and overlapping their neighboring patches by one pixel to later specify $\psi($.$) in the MRF network.$

\subsection{Patch Candidates Selection}

A fixed amount of patch candidates for each node is selected in advance so that we do not have to consider thousands of patches available in the database iteratively during the inferring process. The number of patch candidates is set proportional with the number of training images in the database, e.g., if we used 100 training images, 100 patches from the total number of patches would be selected as candidates.

Patch candidates for each nodes were selected based on $\phi($.$) and$ $\lambda($.) probability values. Training patch with higher pixel value (pattern) similarity and nearer position (closer facial parts) to an observed input patch has a higher possibility to be selected. To adjust the preference between $\phi($.$) and \lambda($.$) , the method apply a$ weighting factor, $\alpha$ as

$$
\begin{aligned}
P_{C}(X \mid Y)= & \prod_{i j} \phi[X(i, j), Y(i, j)]^{\alpha} \\
& \times \prod_{i j} \lambda[X(i, j)]^{(1-\alpha)}
\end{aligned}
$$

The value of $\alpha$ is set between zero and one. Hence, if $\alpha$ is one, the function would not take into account $\lambda($.$) , which means this is the$ same as that with Freeman et al.'s method. The lower the $\alpha$ value, the higher its dependency on facial parts' compatibility function.

\subsection{Super-resolution Process}

The aim is to find the best set of $\mathrm{X}($.$) . By only using the limited$ number of selected patch candidates, $\mathrm{P}(\mathrm{X} \mid \mathrm{Y})$ would typically be at optimum value. Figure 6 outlines the super-resolution process, i.e., deriving an output HR image from a single input LR image. We initially upscale the input LR image into a targeted size of HR image, and then extracted its texture. We divided it into patches and find the most similar patches in database Po, where those corresponding patches from database Pc were placed together to build a base image for the MRF learning model. This image would look uneven or blocks since the chosen patches would not yet be compatible with their neighboring patches. Here, we applied an iteration process so that the chosen patches would be

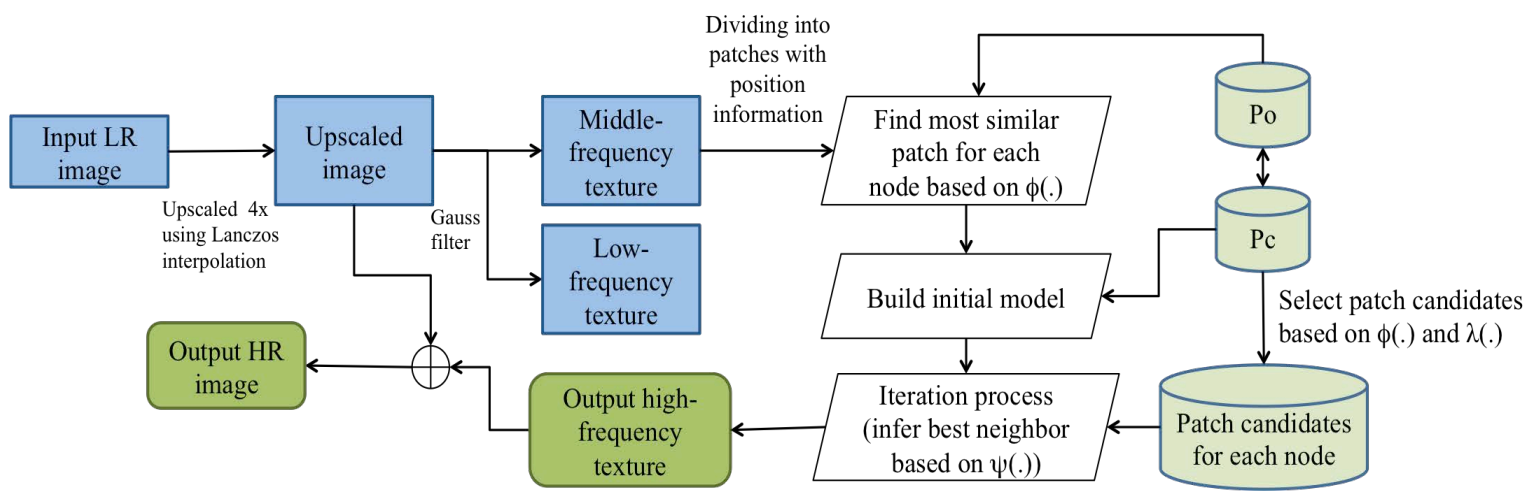

Figure 6. Super-resolution process. 
compatible with their neighboring patches.

According to Equation 7, we replace $\mathrm{X}^{\mathrm{C}}$ with the chosen candidates alternately to find the best $\mathrm{X}(.) . \mathrm{P}_{\mathrm{C}}(\mathrm{X} \mid \mathrm{Y})$ is the known probability value from Equation 6, which is different for each patch candidates.

$$
\begin{aligned}
P(X \mid Y)= & \prod_{(u, v) \in N B(i, j)} \psi\left[X^{C}(i, j), X(u, v)\right], \\
& \times P_{C}(X \mid Y)
\end{aligned}
$$

We replace the initial chosen patches with the best patches that are compatible with the neighboring patches (patches with the best $\psi($.$) values) among the patch candidates on each node. The$ first iteration is done when all nodes have been processed. This resulting image is smoother than that from the initial image model. We carry out the same procedure iteratively until the $\mathrm{P}(\mathrm{X} \mid \mathrm{Y})$ value shows no significant improvement. Finally, selected HR patches on each node were stitched together to form an estimated texture (high-frequency component) image, and then we combined them with the previously upscaled image to obtain the final output HR image.

\section{PROPOSED METHOD}

The previous face image SR method relies on patch-position's coordinate during patch candidates selection process, so patches from face images in database must be divided in a manner of a two-dimensional grid map, where if patch size was $5 \times 5$, patches were divided by interval of five pixels horizontally and vertically. This cutting method caused some fine texture patterns unavoidably being neglected, compare to typical one-pixelinterval cutting. Therefore, we proposed a face image SR method that also considers facial parts during patch estimation, and on the other hand compatible with one-pixel-interval cutting approach.

Even a set of normalized face images was used, yet each face has different size or shape of face feature, i.e. small or big eye, full or thin lip, pointed or rounded nose tip and so on. By using onepixel-interval fine cutting method, we can increase accuracy of texture similarity since more detailed patches were observed.

We proposed new patch-searching algorithms that independent from relying on patch-position information to estimate their facial parts. The algorithms use only information of test image's patch position to indicate the targeted position, and then restricted a certain range of searching area nearby the currently targeted position or facial part.
Figure 7 illustrates the searching method to select patch candidates for each node. Let $(a, b)$ is test image's current patch position, we pinpoint the exact same position onto training images in database. Then, we set a certain range of searching area, $(2 s+1)^{2}$ around the pinpointed position. We raster-scanning patches available within the specified search area in each training images through the pixels from left to right, top to bottom (scan line by scan line) to select a set of 100 most similar patches as patch candidates for each nodes. If $s$ is 10 , searching area will be within $21 \times 21$ pixels of perimeter around the pinpointed position of node, giving 441 patches to be considered from each training face image.

Since facial parts compatibility function, $\lambda($.$) is not relevant for$ this method, we only observed patch similarity, $\phi($.$) as in$ conventional method (Equation 1) to find patch candidates. Then, we iteratively replace patch for each nodes amongst selected patch candidates to find the best possible set of neighboring patches according to border compatibility, $\psi($.$) .$

\subsection{Results and Discussions}

We demonstrated our proposed method on a set of 30 test LR face images using different size of search area, $(2 s+1)^{2}$, where $s$ is set as $3,5,10,15,20,30$ and 40 . The number of face images available in database (excluded test images) is 100 .

\subsubsection{PSNR assessment}

We observed average PSNRs for 30 resulting HR face images as shown in Table 1, while Figure 8 presents the graph.

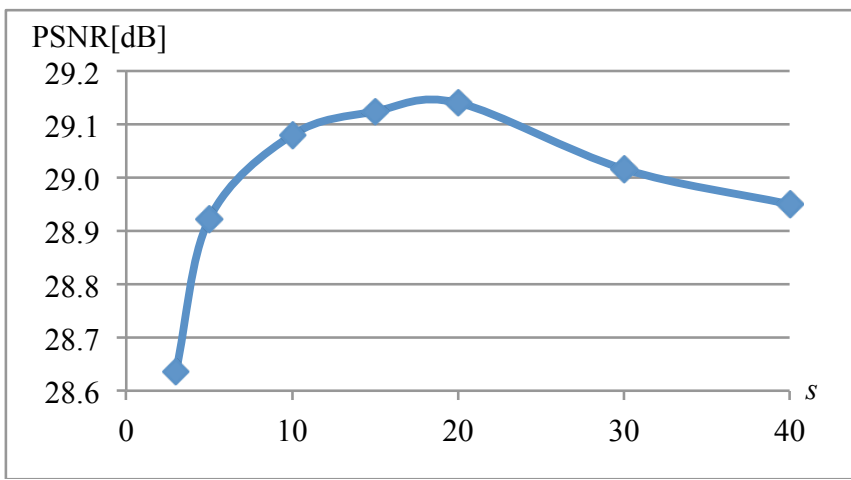

Figure 8. Average PSNR for 30 resulting HR images with different size of search area.

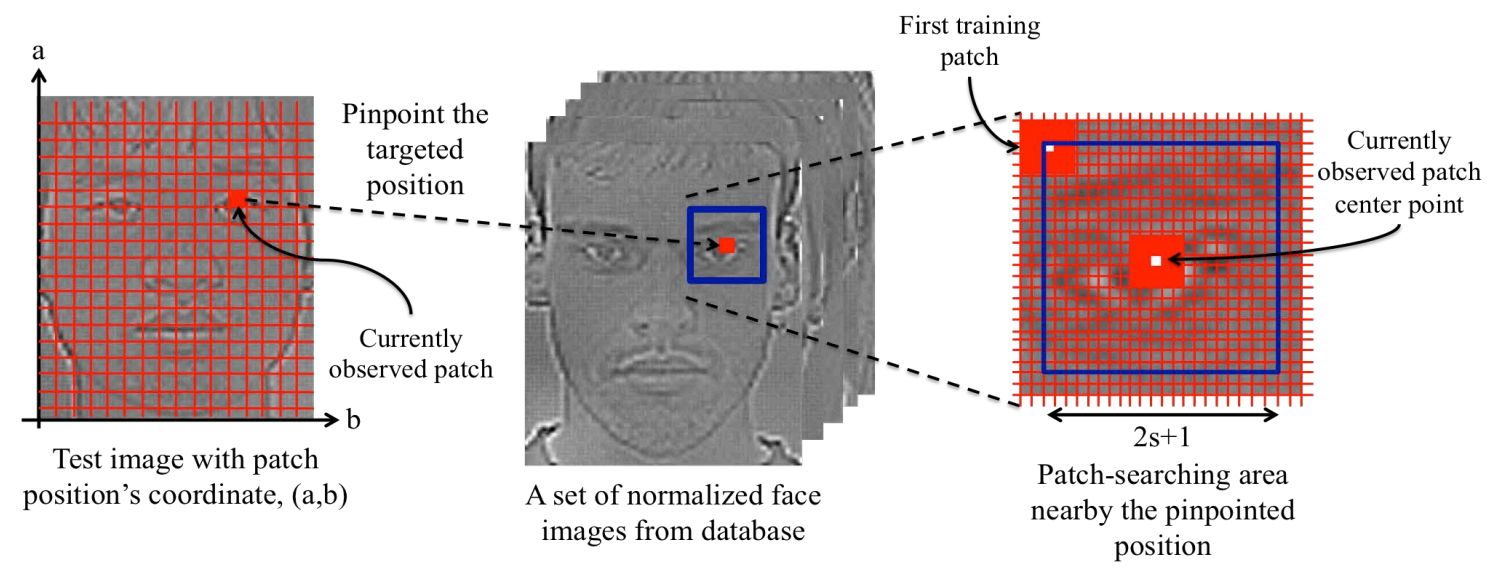

Figure 7. Proposed patch-searching algorithms. 
Table 1. Average PSNR for 30 resulting HR images with different size of search area

\begin{tabular}{|c|c|}
\hline Search area radius, $s$ & $\begin{array}{c}\text { Average PSNR } \\
{[\mathrm{dB}]}\end{array}$ \\
\hline 3 & 28.635 \\
\hline 5 & 28.922 \\
\hline 10 & 29.080 \\
\hline 15 & 29.124 \\
\hline 20 & 29.140 \\
\hline 30 & 29.016 \\
\hline 40 & 28.950 \\
\hline
\end{tabular}

Hypothetically, the wider the search area the better the results, since more number of training patches were observed for selecting patch candidates. However, Figure 8 indicates that the PSNR value decreased as $s$ was larger than 20 . We found that the results were better when a certain range of search area was set, i.e. when $s$ is between 10 and 20 . We conclude that even if we may found much higher similar LR texture (middle-frequency component) from patches available outside the perimeter or far from the targeted position, it do not necessarily give suitable corresponding HR texture (high-frequency component) since they came from different facial parts. Therefore, by restricting search area nearby the targeted facial part, the proposed method manages to find similar LR patches from database that give plausible corresponding HR patches to reconstruct the HR image.

\subsubsection{Computational cost}

Since our method restricted patch-searching area, the number of searched patches during patch-candidates selection was considerably fewer than the previous method. As shown in Table 2 , from a set of 100 training face images available in database, the number of searched patches for each node for proposed method is about one-third (when $s$ is 20) of the number of searched patches in previous method. Furthermore, the proposed method achieves better resulting PSNR than the previous ones.

Table 2. Number of searched patches for previous and proposed method

\begin{tabular}{|l|c|c|}
\hline & $\begin{array}{l}\text { Previous method } \\
\text { (using facial parts } \\
\text { compatibility, } \lambda(.) \text { ) }\end{array}$ & $\begin{array}{l}\text { Proposed method } \\
\text { (using restricted } \\
\text { search area, s=20) }\end{array}$ \\
\hline $\begin{array}{l}\text { Number of } \\
\text { searched } \\
\text { patches for each } \\
\text { node (from 100 } \\
\text { training images) }\end{array}$ & 432,000 & 168,100 \\
\hline $\begin{array}{l}\text { Average PSNR } \\
{[\mathrm{dB}]}\end{array}$ & 28.91 & \multicolumn{1}{|c|}{29.14} \\
\hline
\end{tabular}

The smaller the size of search area, the smaller the number of searched patches, the faster the processing time. From Figure 8, according to the resulting average PSNRs, we can observe that we only need to search within 12,100 patches $(s=5)$ in order to get a resulting HR image that has better or similar quality as the resulting HR image from previous method, i.e. 28.91[dB]. That was approximately 1/35 times the number of searched patches in previous method. In other words, the proposed method has not only improved searching algorithms and given better results yet on the other hand, greatly reduced computational cost.

We compared the proposed method processing time with the previous method's. Table 3 presents experiment environment for the process.

Table 3. Experiment environment

\begin{tabular}{|c|c|}
\hline OS & Windows 7 Home Premium SP1 \\
\hline CPU & Intel Core i5 3.2GHz \\
\hline Memory & CGB \\
\hline Language & C+ \\
\hline Software & Visual Studio 2012 \\
\hline
\end{tabular}

We demonstrated the previous method, where $\alpha$ is set as 0.7 as recommended in [13] to reconstruct an HR image. While for the proposed method, we set $s$ as 20 . We calculated the processing time for three stages of operation, which are database construction, patch-candidates selection and iteration process, for the sake of comparison.

Table 4. Comparison of computational time between previous and proposed method

\begin{tabular}{|c|c|c|}
\hline \multirow{2}{*}{ Process } & \multicolumn{2}{|c|}{ Computational time[s] } \\
\cline { 2 - 3 } & $\begin{array}{c}\text { Previous } \\
\text { method }\end{array}$ & $\begin{array}{c}\text { Proposed } \\
\text { method }\end{array}$ \\
\hline Database construction & 71.47 & 49.91 \\
\hline Patch candidates selection & $\mathbf{6 0 7 . 5 2}$ & $\mathbf{5 2 . 8 3}$ \\
\hline Iteration & 2.44 & 2.66 \\
\hline Whole process & 918.62 & 141.44 \\
\hline
\end{tabular}

Table 4 shows the record of computational time for each stage for previous and proposed method. In comparison, computational time for patch-candidates selection process shows huge different between both methods. We successfully reduced the computational time by approximately $90 \%$ by using the proposed patch-searching algorithms for patch-candidates selection. The computational times for other stages show insignificant different between both methods since those stages are unassociated with the proposed patch-searching algorithms.

\section{CONCLUSIONS}

This paper proposed an improved method for face image superresolution that takes facial parts into consideration. Previous method utilized a set of normalized face images as database, and employed patch's position information to estimate facial parts' compatibility. Since the method relies on patch's coordinate to estimate the facial parts, patches from images in database were divided in a manner of five or more pixels intervals according to patch size. This way of patch-division caused some detailed texture patterns may have been overlooked, compare to pixel-bypixel patch-division. Hence, we have improved patch-searching method to be compatible with one-pixel-interval patch division so that more detailed texture or position can be observed. We 
restricted patch-searching area so that patch-candidates are selected from amongst patches around targeted facial parts, and at the same time to cope with the massively increased size of the patch database due to fine division of patches. In other words, we have liberalized the way of patch-division to obtain much better details of texture, but on the other hand restricted patch-searching area to take facial parts into consideration. Although less number of training patches was observed due to restricted search area, the proposed method achieves better resulting PSNRs compare to the previous method, thanks to the fine way of patch-division. On top of that, we have reduced computational time by $90 \%$ since the number of searched patches has been considerably reduced. In summary, the proposed method obtains better resulting PSNR of HR image than the previous method's with faster processing times.

\section{REFERENCES}

[1] He, H., and Siu, W.-C. 2011. Single image super-resolution using Gaussian process regression. In Proc. IEEE Int. Conf. Comp. Vision and Pattern Recog. (CVPR2011), 449-456.

[2] Baker, S., and Kanade, T. 2002. Limits on super-resolution and how to break them. IEEE Trans. on Pattern Analysis and Machine Intelligence (2002), vol. 24, no. 9, 1167-1183.

[3] Siu, W.-C., and Hung, K.-W. 2012. Review of image interpolation and super-resolution. In Signal \& Information Process. Assoc. Annual Summit and Conf. (APSIPA ASC, 2012), IEEE, 1-10.

[4] Sabyasachi, M. 2017. Single-Image Super-Resolution Techniques: A Review. International Journal for Science and Advance Research in Technology (April 2017), vol 3, no. 4, 271-283.

[5] Park, S. C., M. K. P. and M. G. Kang, "Super-Resolution Image Reconstruction: A Technical Overview," IEEE Signal Processing Magazine (2003), vol. 20, 21-36.
[6] Lui, S. F., Wu, J. Y., and Mao, H.-S. 2007. Learning-based super-resolution system using single facial image and multiresolution wavelet synthesis. Lect. Notes Comp. Sci. 4844 (2007), 96-105.

[7] Jiji, C. V., and Chaudhuri, S. 2006. Single-frame images super-resolution through contourlet learning. EURASIP Journal on Advances in Signal Processing (2006), 1-11.

[8] Wu, W., Liu, Z., Gueaieb, W., and He, X. 2011. Singleimage super-resolution based on Markov Random Field and contourlet transform. SPIE Journal of Electronic Imaging (2011), vol. 20, no. 2, 023005 .

[9] Yang, J., Wang, Z., Lin, Z., and Cohen, S. 2012. Coupled Dictionary Training for Image Super-Resolution. IEEE Trans. on Image Process. (2012), vol. 21, no. 8, 3467-3478.

[10] Freeman, W.T., and Pasztor, E.C. 2000. Markov Networks for Super-resolution. In Proc. 34th Ann. Conf. Info. Sciences and Systems (CISS 2000), Dept. Electrical Eng., Princeton Univ..

[11] Freeman, W.T., Pasztor, E.C., and Carmichael, O.T. 2000. Learning Low-Level Vision. International Journal of Computer Vision, vol. 40, no. 1, 25-47.

[12] Freeman, W.T., Jones, T.R., and Pasztor, E.C. 2002. Example-based super-resolution. IEEE Comp. Graphics Appl., vol. 22, no. 2, 56-65.

[13] Hamdan, S., Fukumizu, Y., Izumi, T., and Yamauchi, H. 2017. Example-based Face Image Super-resolution Taking into Consideration Correspondence of Facial Parts. IEEJ Trans. on Electronics, Info. and Systems, vol. 12, no. 6. 